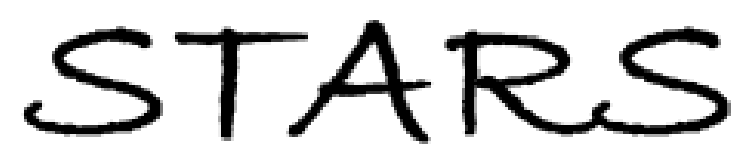

University of Central Florida

STARS

$1-1-2004$

\title{
Architecture and sparse placement of limited-wavelength converters for optical networks
}

\author{
Mounire El Houmaidi \\ Mostafa A. Bassiouni \\ University of Central Florida \\ Guifang Li \\ University of Central Florida
}

Find similar works at: https://stars.library.ucf.edu/facultybib2000

University of Central Florida Libraries http://library.ucf.edu

This Article is brought to you for free and open access by the Faculty Bibliography at STARS. It has been accepted for inclusion in Faculty Bibliography 2000 s by an authorized administrator of STARS. For more information, please contact STARS@ucf.edu.

\section{Recommended Citation}

Houmaidi, Mounire El; Bassiouni, Mostafa A.; and Li, Guifang, "Architecture and sparse placement of limited-wavelength converters for optical networks" (2004). Faculty Bibliography 2000s. 4332.

https://stars.library.ucf.edu/facultybib2000/4332

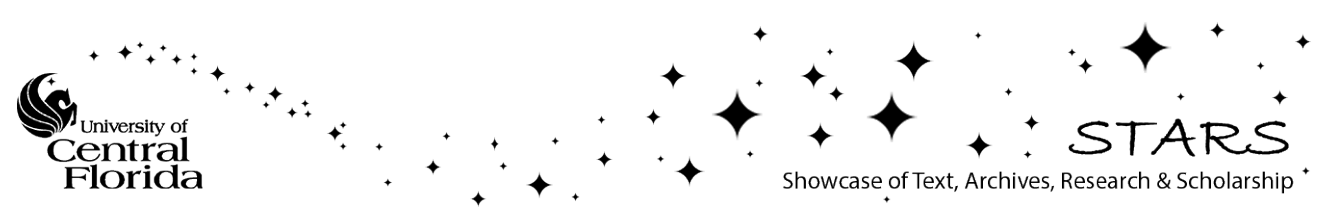




\section{Architecture and sparse placement of limited- wavelength converters for optical networks}

\author{
Mounire El Houmaidi \\ Mostafa A. Bassiouni \\ University of Central Florida \\ School of Electrical Engineering \& Computer \\ Science \\ Orlando, Florida 32816 \\ E-mail: elhoumai@cs.ucf.edu
}

Guifang Li, MEMBER SPIE

University of Central Florida

School of Optics

Orlando, Florida 32816

\begin{abstract}
Equipping all nodes of a large optical network with full conversion capability is prohibitively costly. To improve performance at reduced cost, sparse converter placement algorithms are used to select a subset of nodes for full-conversion deployment. Further cost reduction can be obtained by deploying only limited conversion capability in the selected nodes. We present a limited wavelength converter placement algorithm based on the $k$-minimum dominating set ( $k$-MDS) concept. We propose three different cost-effective optical switch designs using the technologically feasible nontunable optical multiplexers. These three switch designs are flexible node sharing, strict node sharing, and static mapping. Compared to the full search heuristic of $O\left(N^{3}\right)$ complexity based on ranking nodes by blocking percentages, our algorithm not only has a better time complexity $O\left(\Re N^{2}\right)$, where $\Re$ is the number of disjoint sets provided by $k$-MDS, but also avoids the local minimum problem. The performance benefit of our algorithm is demonstrated by network simulation with the U.S Long Haul topology having 28 nodes ( $R$ is 5 ) and the National Science Foundation (NSF) network having 16 nodes ( $\Re$ is 4). Our simulation considers the case when the traffic is not uniformly distributed between node pairs in the network using a weighted placement approach, referred to as $k$-WMDS. From the optical network management point of view, our results also show that the limited conversion capability can achieve performance very close to that of the full conversion capability, while not only decreasing the optical switch cost but also enhancing its fault tolerance. () 2004 Society of Photo-Optical Instrumentation Engineers. [DOI: 10.1117/1.1629122]
\end{abstract}

Subject terms: limited wavelength conversion; all-optical wavelength-division multiplexing simulation and modeling; optical cross-connect design; wavelength converter placement; minimum dominating set.

Paper 030286 received Jun. 16, 2003; revised manuscript received Jul. 17, 2003; accepted for publication Jul. 18, 2003. This paper is a revision of a paper presented at the SPIE conference on Optical Transmission Systems and Equipment for WDM Networking II, September 2003, Orlando, Florida. The paper presented there appears (unrefereed) in SPIE Proceedings Vol. 5247.

\section{Introduction}

The performance of a wavelength routed optical network (WRON) is directly dependent on the capability of its optical cross-connects (OXCs) to enable wavelength conversion. ${ }^{1}$ A wavelength converter can transfer an optical signal on one wavelength at an input port to another wavelength at an output port. Not only has the cost of these devices and OXC complexity made it expensive to have full-wavelength conversion capability at every node, but also wavelength conversion does not always minimize the network's connection blocking. For instance, with a fully connected network, any connection request requires a 1-hop path from source to destination. Wavelength converters are not required at all to improve the blocking percentage of such dense network. Wavelength conversion must therefore be used judiciously and should be placed in nodes that maximize the overall network performance, taking into consideration the network topology and the traffic flowing through its nodes.
The placement of wavelength converters in all-optical wavelength-division multiplexing (WDM) networks has been an active research topic in the recent years. This is due to the fact that the optimal placement of converters in WRON of a general topology is NP-hard. ${ }^{2}$ Most known algorithms apply to special topologies ${ }^{1}$ or are based on placement heuristics. ${ }^{2-8}$ We review these placement schemes and the reported results in Sec. 2.

In this paper, we extend our $k$-minimum dominating set $\left(k\right.$-MDS) algorithm ${ }^{9}$ to the case of limited wavelength conversion using three OXC designs. In Ref. 9, we applied the $k$-MDS concept to the case of sparse placement of fullwavelength conversion. The algorithm uses the idea of having full-wavelength conversion capability at the nodes receiving higher traffic in the network. Consequently, the overall network blocking percentage is decreased, the wavelength reuse is increased and higher loads can be supported. These nodes, as dominating nodes, have to be closer to the nodes without full-wavelength conversion to increase the throughput. For the case of $k$ equals 1 , a one-minimum 
dominating set $D$ is one in which each vertex is either in $D$ or adjacent to some vertex in $D$.

The $k$-MDS problem is NP-complete, ${ }^{10,11}$ and it is related to the traveling salesperson problem requiring approximating heuristics. Our extension of $k$-MDS to the case of limited wavelength conversion is based on dividing the nodes of the network into $\mathfrak{R}+1$ disjoint sets based on the domination factor. To place a wavelength converter given that $Z$ wavelength converters are already suboptimally placed, one node is selected from each set based on the experienced blocking performance. We simulate the network with the wavelength converter placed in each of the selected $(\Re+1)$ nodes separately; the $(Z+1)$ 'th wavelength converter will be placed at the node that gives the lowest overall network blocking performance. Our simulation considers also the case when the traffic is nonuniformly distributed between node pairs, as in Ref. 12 .

Our approach minimizes the search space and enables better results than the fullsearch algorithm previously proposed in the literature. ${ }^{3,4}$ The local minimum problem reported in Ref. 3 is eliminated since the $k$-MDS scheme solves this minimization problem by finding a solution taking into consideration the traffic flowing through the nodes. We show via simulation that our placement algorithm for limited wavelength conversion is cost effective and improves the utilization of the network. To our knowledge, our work is the first attempt to apply the MDS approach to the design and engineering of optical networks in general and to the limited wavelength conversion placement in particular.

The remainder of this paper is organized as follows. Section 2 reviews related studies on wavelength converters placement. Section 3 describes the considered OXC switch designs for flexible node sharing, strict node sharing, and static mapping. Section 4 introduces the extension of $k$-MDS for the limited wavelength conversion case. Section 5 presents our simulation model for limited wavelength conversion placement. We show its performance using the U.S Long Haul topology under uniform traffic. In section 6, we consider the case when the traffic is not uniformly distributed between node pairs. Performance results are also presented in this section using the National Science Foundation Network (NSFNET) topology. We make concluding remarks in Sec. 7.

\section{Related Work}

The introduction of wavelength conversion (WC) into wavelength routed optical networks enables a significant reduction of the blocking probabilities and an increase of the overall throughput. The conversion capability also increases the cost and complexity of the OXCs. Converters must therefore be used judiciously and must be placed in nodes that maximize performance improvement. Hence there have been a number of studies to investigate sparse and limited wavelength converter placement in optical networks.

In Ref. 1, the authors introduced the conversion density factor $q$ of the network and an analytical model taking into consideration the correlation of wavelength usage between adjacent links. On three network topologies (ring, meshtorus, hypercube), the reported results show that the uniform placement of sparse WC (only selected nodes equipped with full conversion capability) is cost effective and can achieve most of the benefits of full conversion at every node of the network. The authors reported that the uniform placement technique works well for topologies with low connectivity such as rings but is not suitable for general topologies. This is due to the fact that the scheme does not take into consideration the topology of the network, the connectivity of each node and the expected traffic load.

The study in Ref. 2 explores the heuristic of placing full wavelength conversion at nodes with high nodal degree. A network simulation is used to show the benefits of this placement heuristic. The simulation model uses an auxiliary graph, with $M$ nodes, constructed based on the physical topology of the network. Note that $M$ is the product of $N$, the number of nodes in the network, and $W$, the number of wavelengths. The arcs are labeled with channel costs and conversion costs. Dijkstra's algorithm is applied for each connection request. The model considers a dynamic light path establishment. The used arcs are removed to reflect the wavelength usage in the network. When the connection is terminated, the graph is updated to reflect the newly released resources. The simulation results show that it is cost effective to place full WC at high degree nodes; and that wavelength converters provide a significant gain in sparse networks but very little improvement in highly dense networks.

The authors in Ref. 3 explored optical switch designs with limited number of wavelength converter units at each node. The designs implement the limited WC with sharednodal switch design using tunable optical multiplexers. The OXC can contain share-per-node wavelength converter units, or share-per-link wavelength converter units that are shared by the incoming circuits. This type of limited conversion switch has the potential of achieving most of the benefits of a full conversion-capable switch at a much lower cost. A heuristic of complexity $O\left(N^{3}\right)$ was introduced in Ref. 3 for placement of individual limited wavelength converter units in the nodes of the optical network.

In Ref. 4, the authors introduced a ranking-based heuristic for limited converter placement. Via simulation, each node is ranked given the experienced blocking performance. Iteratively, the heuristic reshuffles the wavelength converters between nodes with low blocking to nodes with high blocking until there is no improvement in the overall blocking performance. The results indicate that shared-perlink architecture is cost effective and provide a good tradeoff for performance compared to the shared-per-node architecture. The disadvantage of such heuristic is its complexity when considering large networks.

The study in Refs. 5 and 6 shows that routing and wavelength assignment with wavelength conversion capability is an NP-complete problem. The OXC enables wavelength converters to be shared at the node level or between links. It also requires tunable optical multiplexers that make the design more expensive. A routing algorithm is introduced, similar to Ref. 2, with a reported time complexity of order $O\left(N^{4} \times W^{2}\right)$ without including the number of incoming connection requests to the network in the analysis.

The authors in Ref. 7, instead of using time-consuming simulations, presented an analytical model for arbitrary topologies and traffic loads. It is assumed that each node has 
a converter with a probability $q$. An analytical model is introduced to take into consideration the dependence and correlation of wavelength usage between adjacent links. It is assumed that the wavelength usage on a link is dependent only on the usage on an adjacent link. The authors reported that the model provides accurate blocking probabilities compared to simulation results, but it is not tractable for networks with high diameter.

In Ref. 8, the authors focused on shared-per-link design, where each outgoing link has a dedicated converter bank that is only used by connection passing through this particular link. The complexity of such design, based on tunable multiplexers, is cost effective compared to the sharedper-node OXC design. The placement problem is modeled as an integer linear program (ILP) in which the objective function is to maximize the total amount of traffic passing through the network. An acyclic auxiliary graph is built in the same way as in Ref. 2 and the Bellman-Ford algorithm is used to find the shortest path between a source and destination to establish a connection request. The time complexity of the approach is dependent on the size of the network $N$, the number of wavelength per link $W$, and the total number of simulated connection requests $C$. The time complexity of the approach is order $O\left(C \times N^{4} \times W^{2}\right)$.

The study in Ref. 13 introduces a novel placement for full WC for minimal wavelength usage. This is achieved by causing the number of wavelength required to be equal to the maximal link load. The scheme achieves loadwavelength assignability and it is based on splitting a general topology network into simpler subgraphs such as paths and spiders, as in Ref. 14. The reported results show that the approach combines the problem of finding the minimum wavelength needed and the problem of full WC placement.

The authors in Ref. 15 focus on survivability, load balancing, and capacity constraints as criteria for WC placement for the static routing wavelength assignment (RWA) problem. The scheme places a full WC at the node with the highest transit load. To avoid the network traffic becoming unevenly distributed, a conversion cost function is used to enable backtracking so that the proposed heuristic can remove a WC if it is not required in the final placement. The proposed algorithm reduces the number of wavelengths used by $21 \%$ compared with the approach in Ref. 16, which uses higher objective functions for states where capacity constraints are violated.

In Ref. 17, a path-metric-based algorithm is used for full WC placement. A weighting factor (WF) enables the ranking of all the nodes and the placement decision is based on the computed values (nodes with higher WF should have full WC). The WF depends on the number of hops between nodes and the interference length taking into consideration the paths that are sharing one or more links with the considered path. The approach applies to static routing when traffic requests are known in advance and does not take into consideration the routing scheme and wavelength assignment.

The study in Ref. 18 considers distributed algorithms minimizing the number of WCs when establishing a connection on a given path. The approach is extendable to sparse WC and limited WC by applying different levels of aggressiveness in locking wavelength during path establish- ment. The reported results indicate that since wavelength availability is dynamic, adaptively assigning the wavelength to use is more crucial than the selection of the route for path establishment.

Some studies investigated other solutions for wavelength contention in WDM networks. The authors in Refs. 19 and 20 explore limited-range WC as a cost-effective solution instead of full WC. The heuristic in Ref. 19 is based on ranking the nodes by nodal degree. An auxiliary graph is built to capture the wavelength available per link, as in Ref. 2. In Ref. 20, an analytical model is used to estimate the network performance for unidirectional rings and mesh-torus topologies. The reported results suggest that with only $50 \%$ of the full WC range, the same the blocking performance can be achieved as with full range conversion. The study in Ref. 21 envisions that if certain nodes are capable of full WC, broadcasting can be supported in WDM networks. The heuristic is based on the color covering and the vertex color-covering problems; and it achieves the optimal solution $52 \%$ of the time with an average performance ratio of 1.169 .

In what follows we describe in detail the considered switch designs enabling limited wavelength conversion and we introduce our placement heuristic. Our simulation results will cover the case of uniform distribution of traffic between (source, destination) pairs and also the case when the traffic is not uniformly distributed between node pairs for the NSFNET and the U.S. Long-Haul topologies.

\section{OXC Switch Design with Limited WC}

Full WC at a selected set of nodes improves the blocking performance of the network. This is referred to as the sparse full WC. Further cost reduction can be obtained by deploying only a limited number of wavelength converter units at each selected node. ${ }^{3,4}$ This is referred to as the limited WC. The overall network performance achieved could be similar to the placement of sparse full WC. This is due to the fact that at any given node only a portion of the WC capability is used at any given time.

All of the proposed designs in Refs. 2 to 7 use tunable multiplexers that do not yet exist and will not be available in the near future due to fundamental limitation of light propagation. Our optical switch designs are ideally suited for the case when fiber capacity or the size of the OXC is overdesigned for future expansions so that there are unused ports available. Consequently, the limited WC does not incur any additional cost of switching. In addition to their scalability, our proposed designs ensure that no tunable multiplexers are required as opposed to the previously proposed architectures in the literature.

In this section, we consider the case of limited $\mathrm{WC}$ in the sense that the number of wavelength converter units $C_{\lambda}$ is smaller than $F \times W$ (needed for full conversion), where $F$ is the number of fibers in the considered node, and $W$ is the number of wavelengths per fiber. These $C_{\lambda}$ any-to-any wavelength converter units are shared by all possible light paths that pass through the optical switch for the case of node-sharing designs. We introduce a flexible and a strict implementation of node-sharing OXC design.

We also explore a design where the wavelength converters are statically mapped to an output link. With this simple optical switch design, there are two choices to make. The 


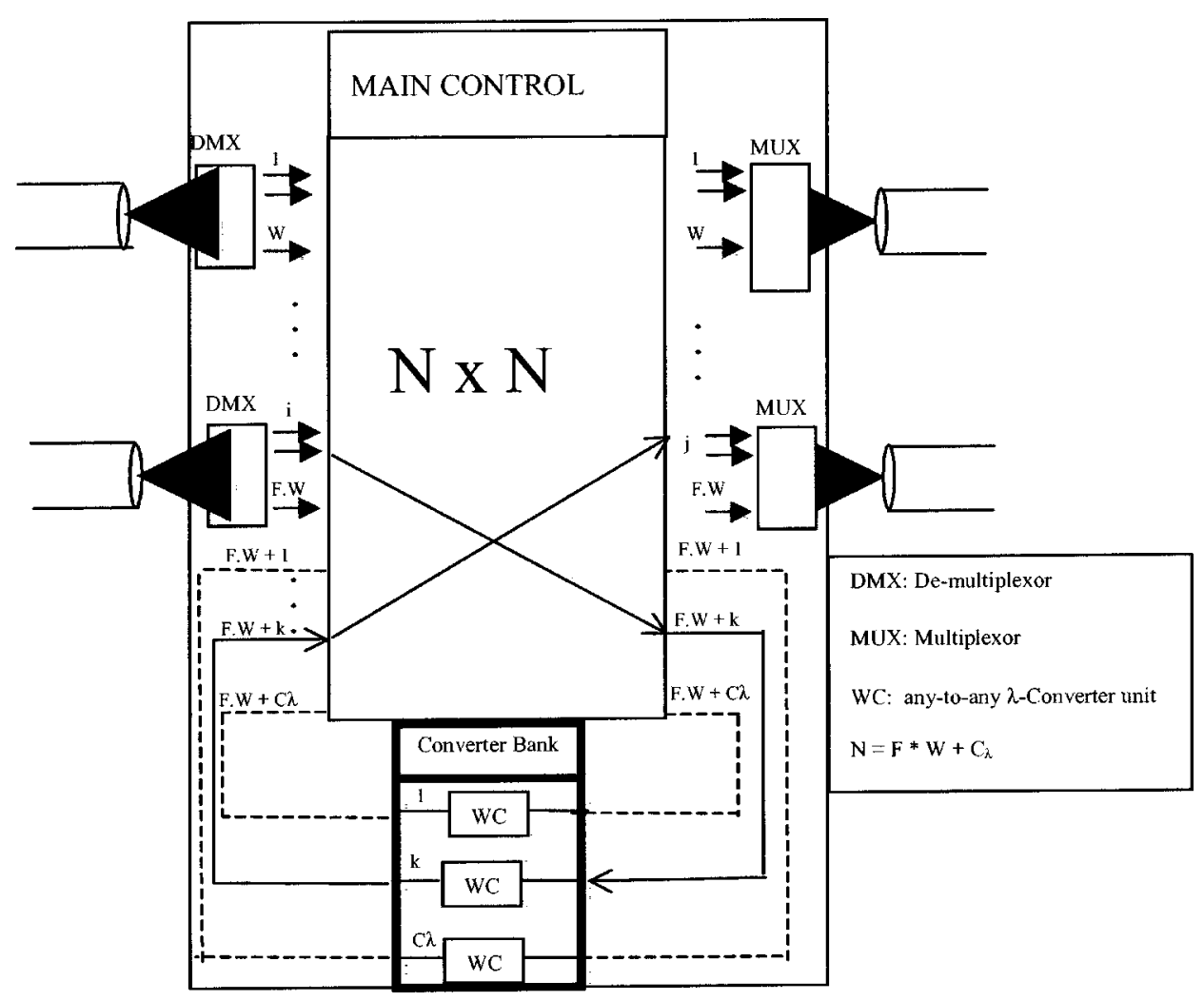

Fig. 1 Flexible node-sharing switch design.

first step is to choose the node for placing the wavelength converter, and the second step is to choose the output link to which this converter will be permanently assigned (statically mapped).

We show via simulation that limited WC achieves most of the benefits of full WC while increasing the fault tolerance. ${ }^{3}$ We also compare our optical switch designs with limited WC, taking into consideration the switch design complexity (cost) and the overall network performance achieved.

\subsection{Flexible Node-Sharing OXC Design}

Our proposed architecture for the flexible node-sharing optical switch with limited WC is shown in Fig. 1. We propose a cost-effective optical switch design using nontunable optical multiplexers. The size of switch node is $N$ $\times N$, where $N \geqslant F \times W+C_{\lambda}$. Any light path that requires WC from input port $i$ to output port $j$ is first routed to one of the output ports, $F \times W+k$, where $1 \leqslant k \leqslant C_{\lambda}$, for WC. The wavelength-converted signal at input port $F \times W+k$ is then be switched to output port $j$.

The flexible node-sharing switch design enables a centralized sharing at the node level of the WC bank. The any-to-any wavelength converters are available to all output links and used by any connection requests.

\subsection{Strict Node-Sharing OXC Design}

The strict node-sharing optical switch with limited WC is shown in Fig. 2. A wavelength converter $C_{\lambda}$ in this design has $W$ subcircuits for fixed WC, and each subcircuit converts any incoming wavelength to one of the $W$ different wavelengths. The wavelength converters are shared among all the outgoing links. However, we assume that once a converter is used for a given output link, it cannot be used for another output link at the same time; but it can be used to convert different wavelengths (up to $W$ ) on the same output link. This assumption is not due to the limitation of the switch fabric, but is made here for easy comparison with the static mapping OXC design described subsequently. When a converter becomes idle, it can be used with a different output link. Note that each conversion subcircuit is specialized to convert to one unique output wavelength. The strict node-sharing design uses simpler conversion units (any-to- $\lambda$ ) as opposed to the flexible nodesharing that uses any-to-any WC units.

In Fig. 2, converter number 1 is used for output link number $F$. Subcircuit 1 , in converter 1 , is used for WC for the connection from input link number 1 to output link number $F$. Subcircuit $W$, in converter 1 , is used for the connection from input link $F$ to output link $F$ at the same time. Converter number $C_{\lambda}$ is idle since all its subcircuits are unused and will be available to an output link if needed.

Note that our placement scheme for the flexible nodesharing design is based on one WC unit to be placed at each step. With the strict node-sharing design, we place one converter containing $W$ subcircuits for WC in each step. Our placement algorithm in either case must select a node to place one single additional WC unit for the flexible nodesharing switch design; and will select a node where to place a converter containing $W$ subcircuits for the strict nodesharing switch design. Consequently, the number of conversion units available in the network will be increased by 1 at 


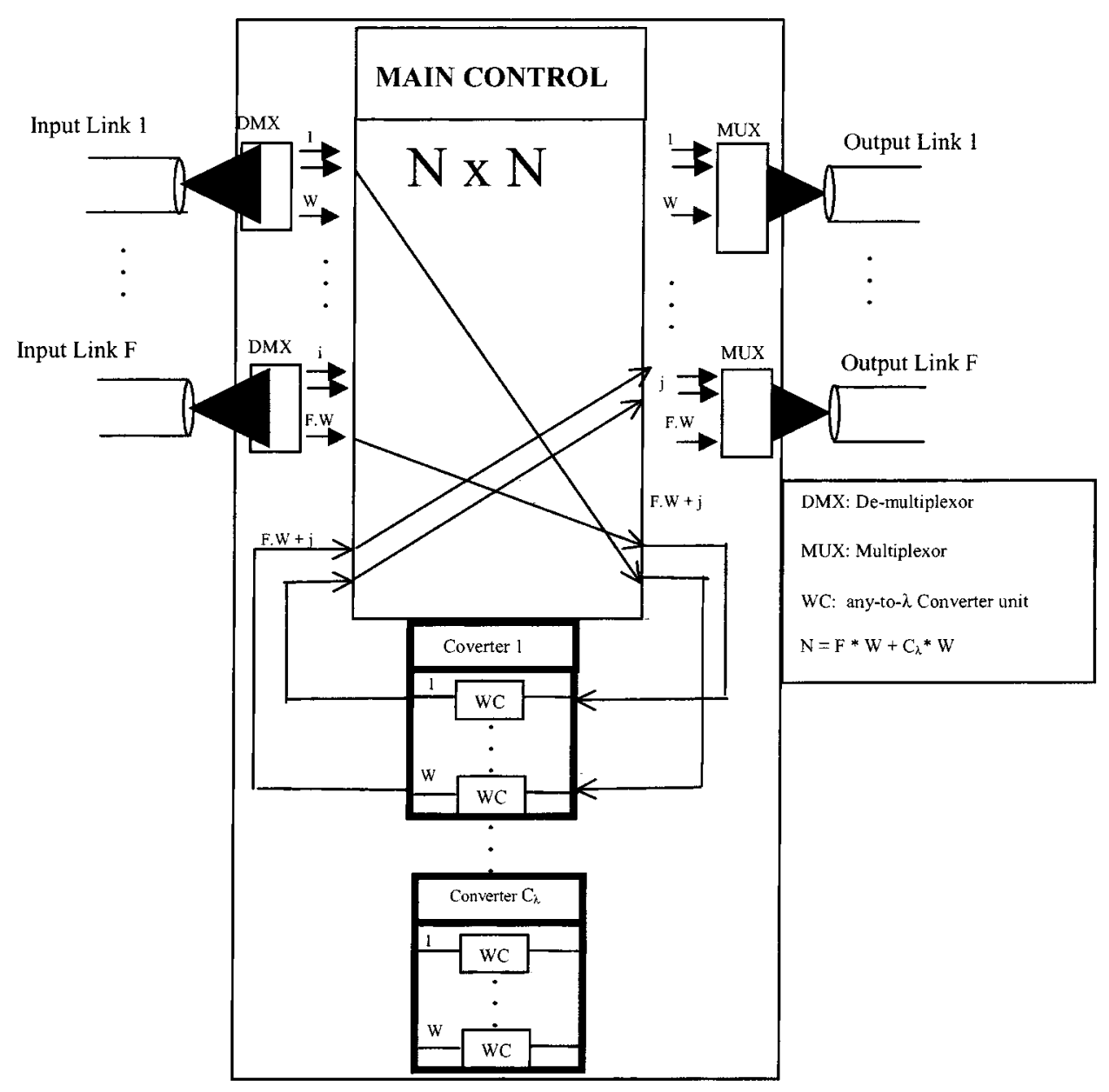

Fig. 2 Strict node-sharing optical switch design.

every step when the flexible node-sharing design is considered and will be increased by $W$ after each step when the strict node-sharing design is studied.

\subsection{Static Mapping OXC Design}

In our static mapping optical switch design with limited WC, a converter is attached to a specific output link on a permanent basis. A wavelength converter $C_{\lambda}$ in this design has $W$ subcircuits to convert to $W$ different wavelengths (similar to the strict node-sharing design). The wavelength converters are not shared among all the outgoing links. A converter is dedicated to a given output link, it cannot be used for another output link, but it can be used to convert different wavelengths (up to $W$ ) on the link it is mapped to. Figure 3 describes the static mapping optical switch design for limited WC. Wavelength converter 1 has $W$ subcircuits dedicated to output link $F$.

The next step is to introduce our limited WC placement algorithm, and apply it to each OXC design and compare its performance against the full search based heuristic introduced in Ref. 3.

\section{$4 \quad k$-MDS Algorithm for Limited-Wavelength Conversion}

In Ref. 9, we formulated this placement problem as a $k$-MDS problem: given a graph $G(V, E)$, determine a set with minimum number of vertices $D \subset V$ such that every vertex in the graph is either in $D$ or is at distance $k$ or less from at least one member in $D$. The members of the set $D$ represent special nodes that act as WC sites within the network.

We developed an approximation algorithm for the $k$-MDS problem that computes the set of master nodes to be equipped with WC capability. ${ }^{9}$ The algorithm provides a suboptimal placement of wavelength converters in an optical network using the topology of the network as an input and independently of the number of wavelengths per link. The algorithm ensures that the resulting set $D$, also referred to as the $k$-MDS set, has the following property: every node $v \in V$ is either in $D$ or is at most $k$ hops away from a node in $D$. The $k$-MDS approach assumes a uniform traffic pattern between each node pair (source $s$ and destination $d$ ).

\subsection{Background and Definitions}

We first present the basic background on $k$-MDS and the related definitions used in our model. Given a graph $G(V, E)$, a $k$-minimum dominating set $k$-MDS in $G$ is a set $D \subset V$ with minimum number of nodes such that every node in the graph is either a member in $D$ or is at distance $k$ or less from at least one member in $D$. The $k$-MDS problem is NP-hard, ${ }^{10,11}$ and heuristic algorithms to solve it (such as the one used in Ref. 9) usually give optimal or 


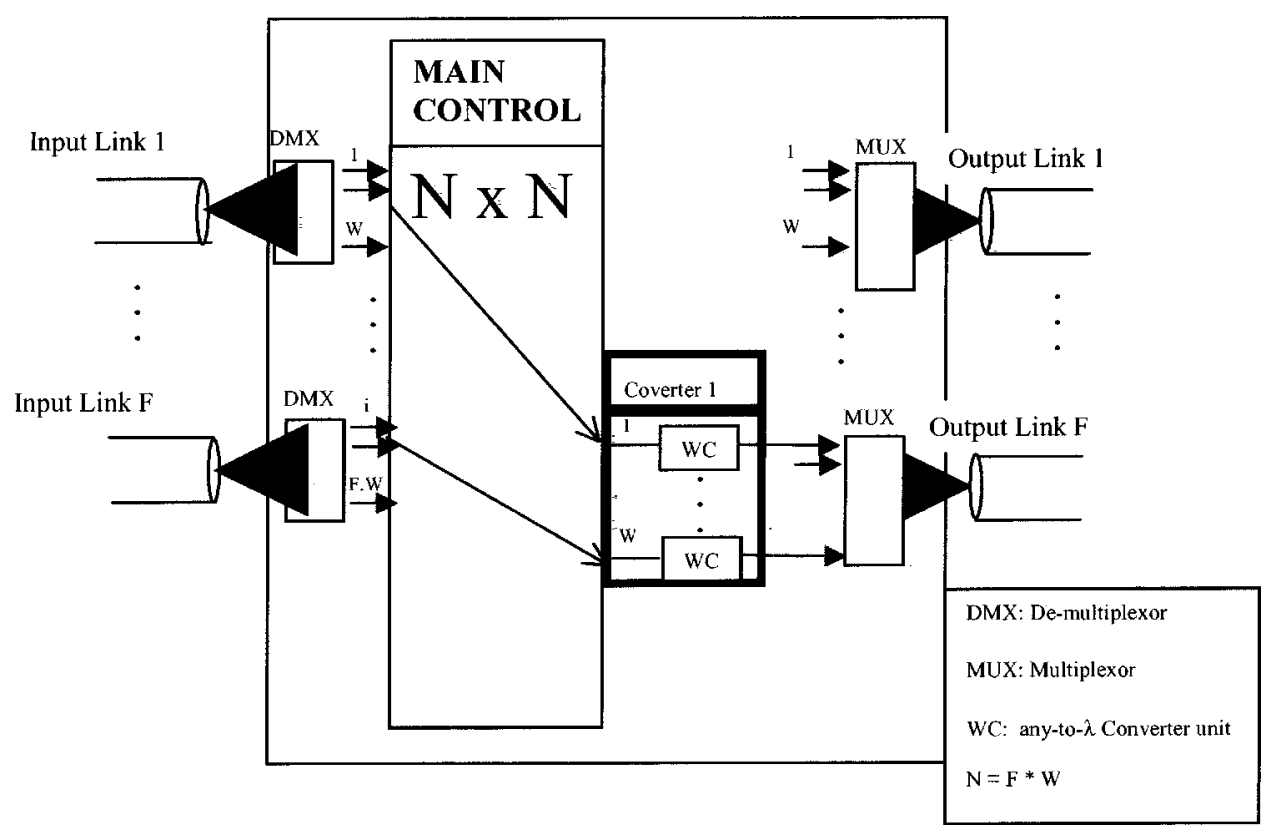

Fig. 3 Static mapping optical switch design.

excellent suboptimal solutions. In our approach, the nodes in $k$-MDS represent special nodes that act as wavelength conversion sites within the network. The index $k$ of $k$-MDS represents the maximum distance allowed between a node in the network and the nearest conversion site. The connectivity of node $v$ based on the index $k$ is measured by $\operatorname{Connect}_{k}(v)$, which is defined recursively as follows:

$\operatorname{Connect}_{0}(v)=\operatorname{Degree}(v)=\operatorname{Cardinality}[\operatorname{Neighbor}(v)]$,

$\operatorname{Connect}_{1}(v)=\operatorname{Connect}_{0}(v)+\sum_{m \in \operatorname{Neighbor}(v)} \operatorname{Connect}_{0}(m)$.

Recursively we define $\operatorname{Connect}_{k}(v)$ as:
$\operatorname{Connect}_{k}(v)=\operatorname{Connect}_{(k-1)}(v)$

$$
+\sum_{m \in \operatorname{Neighbor}(v)} \operatorname{Connect}_{(k-1)}(m),
$$

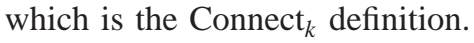

The set Neighbor $(v)$ in the preceding definition denotes the set of nodes sharing a direct link with node $v$. The $\operatorname{Connect}_{k}(v)$ parameter is used in the computation of the $k$-MDS sets. Each node $v$ will vote for the node with the highest $\operatorname{Connect}_{k}(v)$ value over all nodes within $k$ hops from $v$. Figure 4 shows the NSF backbone topology consisting of 16 nodes and 25 links. The marked (double-

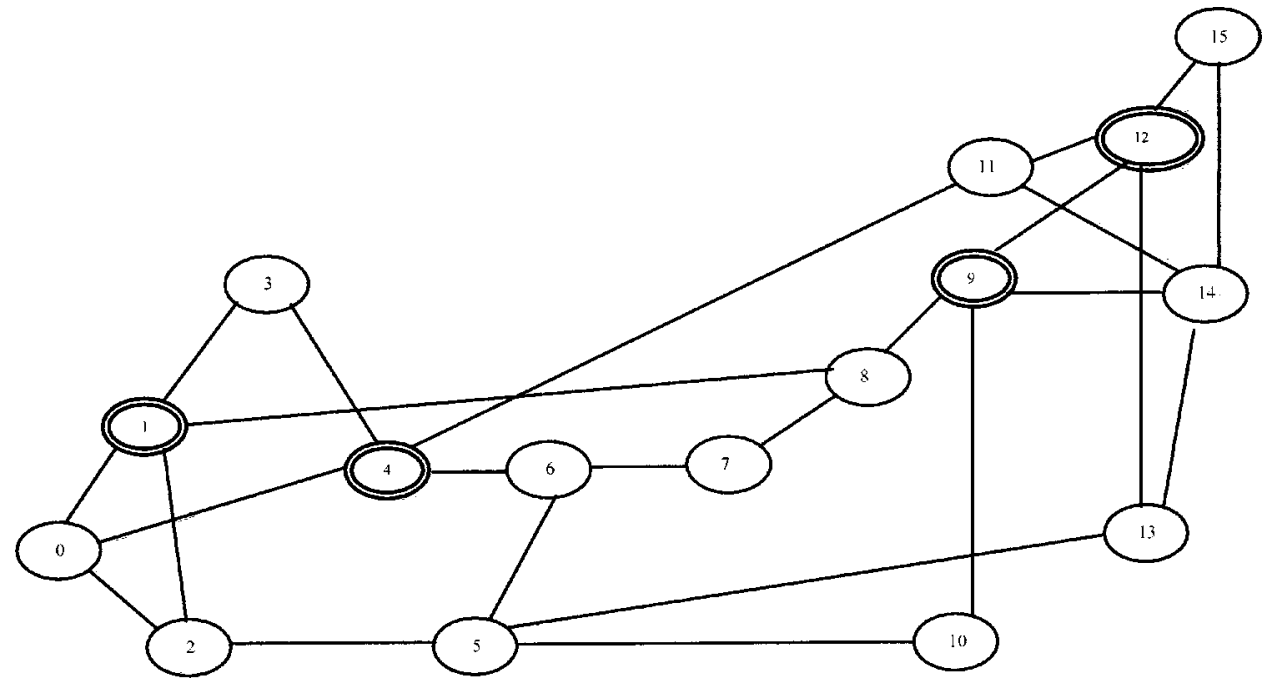

Fig. 4 NSFNET nationwide backbone network. 
circled) nodes in Fig. 4 are the members of the 2-minimum dominating set (2-MDS) set, i.e., every node is either in the 2-MDS set or at least 2 hops away from a member of 2-MDS. Applying the preceding definitions to the NSF backbone topology, we obtain the following:

$1-\mathrm{MDS}=\{1,4,5,6,9,12\}$,

$2-\mathrm{MDS}=\{1,4,9,12\}$,

and$$
3-\mathrm{MDS}=\{12\} \text {, }
$$

Connect $_{0}($ node 0$)=3$,

Connect $_{1}($ node 0$)=14$,

Connect $_{2}($ node 0$)=58$.

We use the NSFNET topology in Sec. 6, where we consider the traffic to be nonuniformly distributed between node pairs in the network.

\subsection{LIMITED Algorithm}

In this section, we propose the LIMITED algorithm for the placement of limited WC based on our $k$-MDS algorithm. Excluding the overhead of the simulation step as was done in Ref. 3, the worst-case complexity of LIMITED is $O(\Re$ $\times N^{2}$ ), where $\Re<N$, as explained shortly. Instead of trying all $N$ possible placements for an individual converter unit as was done in the $F$-SEARCH heuristic, ${ }^{3}$ our algorithm divides the nodes into $(\mathfrak{R}+1)$ disjoints sets based on $k$-MDS and runs the network simulation for the highest blocking node in each of those disjoint sets. The final placement decision will be made based on the node, out of the $(\Re+1)$ selected, that provides the lowest overall network blocking percentage.

Given a network with $Z$ existing converter units already placed semioptimally, our LIMITED algorithm finds the placement for the next $(Z+1)$ 'th converter in the network. As stated earlier, depending on the considered switch design, the converter to be placed is one WC unit for the flexible node-sharing switch design; and it can be a wavelength converter containing $W$ subcircuits when the strict node-sharing is considered. For the static mapping switch design, the algorithm will select a node and then a link to have a statically mapped wavelength converter.

The LIMITED algorithm is described as follows:

1. Compute all $k$-MDS sets, starting at $k=1$, until the last $\mathfrak{R}$-MDS set has one member.

2. Initialize $\mathfrak{R}+1$ disjoint sets: SET $1=\mathfrak{R}-\mathrm{MDS}$, SET $2=(\Re-1)-\mathrm{MDS}-\mathfrak{R}-\mathrm{MDS}$, and SET $3=(\mathfrak{R}-2)$ MDS- $\mathfrak{R}-1)-\mathrm{MDS}$, etc. SET $\mathfrak{R}=1-\mathrm{MDS}-2-\mathrm{MDS}$ and SET $\mathfrak{R}+1$ has the rest of the nodes.

3. Run the network simulation with the existing $Z$ individual converter units in the network.

4. Find the highest blocking node in each set of the $\mathfrak{R}+1$ computed set

5. For each one of the $(\mathfrak{R}+1)$ nodes selected in step 5 , run network simulation with the $(Z+1)$ 'th converter unit placed in that node. For the static mapping case, place converter at the highest blocking link in the node.

6. Place the $(Z+1)$ 'th converter unit in the node with the lowest overall network blocking in step 5 .

Notice that the LIMITED algorithm avoids the greedy full search [examination of all possible combinations to place the $(Z+1)$ 'th converter] as was done in the $F$-SEARCH heuristic. ${ }^{3}$ Instead, it uses a search based on the computed dominating sets $k$-MDS. As we see through simulation results, this makes the LIMITED algorithm more stable and less prone to the local minimum problem reported in Ref. 3. The reported complexity of $F-S E A R C H$ is reported to be $O\left(N^{3}\right)$.

\section{Simulation and Results Under Uniform Traffic}

We compare the performance of each optical switch design using the U.S. Long-Haul topology, ${ }^{2}$ including 28 nodes and 45 links. Each link is a bidirectional fiber with 8 wavelengths $(W=8)$. The connection holding time is exponentially distributed and the traffic is uniformly distributed over all node pairs. When a connection request arrives to the network between a source $s$ and destination $d$, a precomputed shortest path is taken to reserve a wavelength in each link in the path. The wavelength assignment is random and the reservation is done in the forward direction.

Our network simulation is based on a discrete-event model with a time-advance mechanism to the most imminent event to be processed. Each event processing affects the state of the system and the time of occurrence of future events. Our simulation models six different events: an arrival of a connection request to the network, a departure of a reserve control request from a particular node to the next hop node, the event of blocked connection request due to lack of resources, the event of establishment of the connection request at the destination node, the event of termination of an established connection enabling the release of resources, and the event of ending the simulation after $C$ connections processed. Note that $C$ is $10^{6}$ times the considered load of traffic in the network. The load, in Erlangs, is the product of the arrival rate and the average holding time. The simulation takes into consideration the control message processing time $P$ at each node, where $P$ is assumed to be $10 \mu \mathrm{s}$. The propagation delay is estimated based on the kilometric distance between nodes and the speed of light. The hop-based shortest paths between node pairs are computed in advance using Dijkstra's algorithm.

Our extensive simulation tests with randomly generated topologies have shown that the LIMITED algorithm provides excellent placement for nodes with limited WC.

We now apply our algorithm to a realistic topology: the U.S. Long-Haul network ${ }^{2}$ is described in Fig. 5. The double-circled nodes are members of the 2-MDS set.

Our $k$-MDS algorithm ${ }^{9}$ produces the following results for the U.S. Long-Haul network:

$1-\mathrm{MDS}=\{1,3,4,5,8,10,12,15,17,20,22,25,27\}$, 2 -MDS $=\{4,8,12,17,25\}$, every node is at least 2 hops away from a member of 2-MDS, $3-\mathrm{MDS}=\{8,12,17\}$, $4-\mathrm{MDS}=\{12\}$, every node is at least 4 hops away from node 12.

With the U.S. Long-Haul network $\mathfrak{R}$ is 4 , and the computed disjoint sets are

SET $1=4-\mathrm{MDS}=\{12\}$,

SET $2=(3-\mathrm{MDS})-(4-\mathrm{MDS})=\{8,17\}$,

SET $3=(2-\mathrm{MDS})-(3-\mathrm{MDS})=\{4,25\}$,

SET $\quad 4=(1-\mathrm{MDS})-(2-\mathrm{MDS})=\{1,3,5,10,15,20,22$,

27\}, 


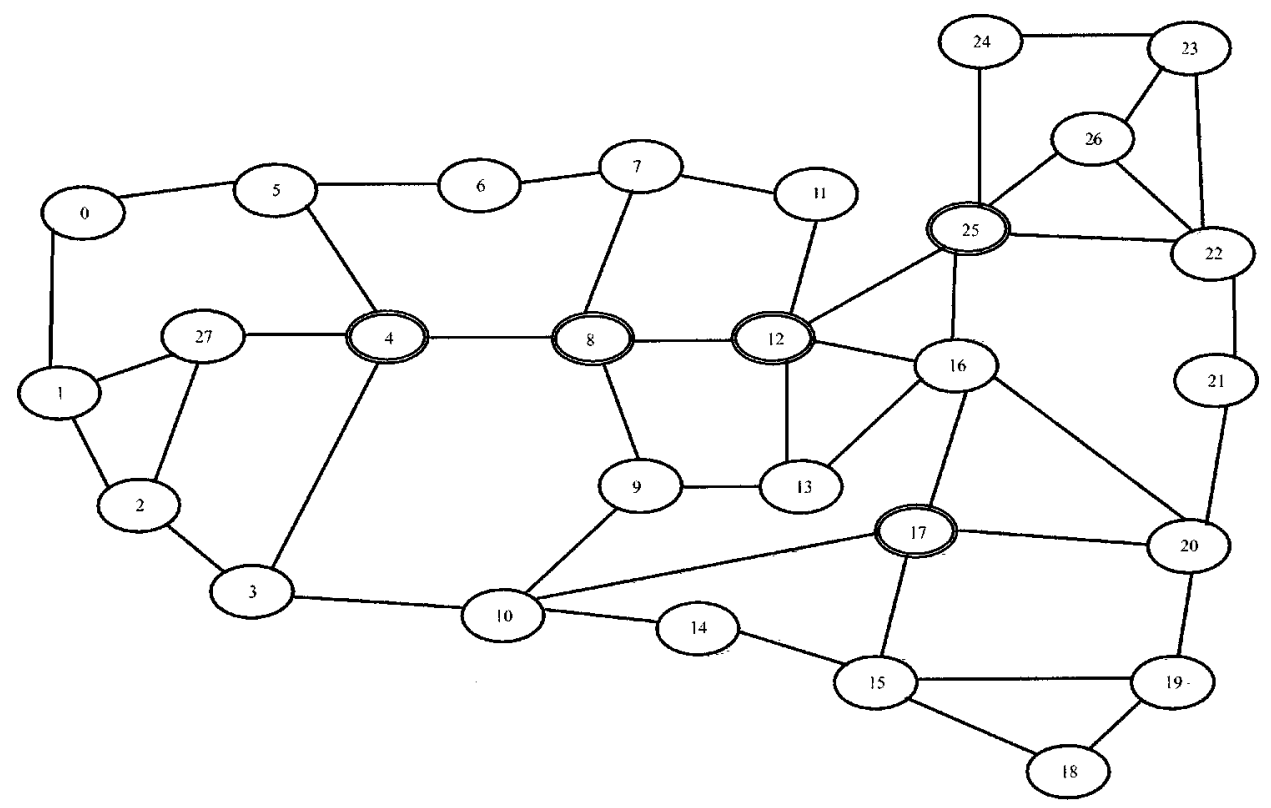

Fig. 5 U.S. Long-Haul network. ${ }^{2}$

SET $5=$ contains the rest of the nodes, i.e., 15 nodes.

Our comparison results of LIMITED and $F$-SEARCH under different traffic loads are presented in Fig. 6 for the flexible node-sharing switch design, in Fig. 7 for the strict node-sharing switch design, and finally in Fig. 8 for the static mapping design. Under a fixed load of 50, we started with no WC and at each step added a unit of conversion following the LIMITED algorithm. We continued to do so until we placed $50 \%$ of the maximum number possible of wavelength converters units, 720 in this case.

For the three proposed switch designs, the results show that the full search of all possible placements by $F$-SEARCH can misexamine a combination of converter placements that reduce the blocking percentage. As it can be seen from the curves of $F$-SEARCH (in Figs. 6, 7, and 8 ), the greedy full search frequently causes the blocking percentage to increase and decrease unexpectedly due to

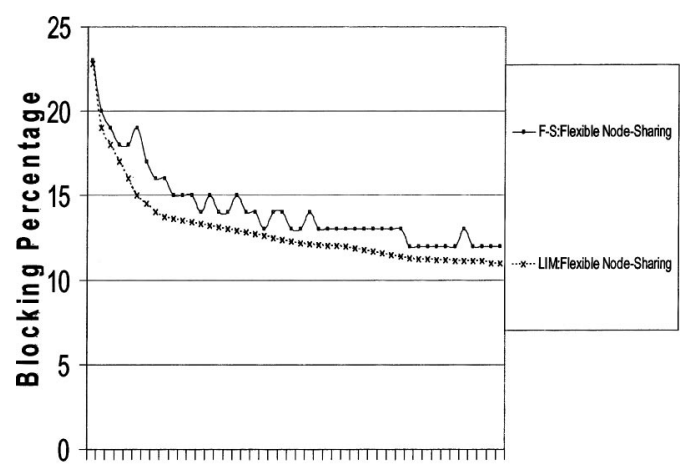

$$
\begin{aligned}
& \text { - } 2^{\circ} 8^{\circ}, 2^{\circ}, 6^{\circ} 2^{\circ} 24^{\circ} 28^{\circ} 32^{\circ} 33^{\circ} \\
& \text { Number of wavelength Converters }
\end{aligned}
$$

Fig. 6 U.S. Long-Haul network using flexible node sharing: LIMITED versus $F$-SEARCH (50 Erlangs, $W=8$ ). the local minimum problem (this problem was also reported in Ref. 3). On the other hand, the LIMITED algorithm is more stable since we base our search on $k$-MDS sets; and it is also faster and less complex.

Figure 9 compares the proposed optical switch designs using the same method. The flexible node-sharing outperforms the other design since it enables all output links to share the WC bank. It fulfills the WC requirements of incoming connection request in a centralized fashion. The flexible node-sharing is suited when 200 or less wavelength converters are to be placed in the network (around $25 \%$ of the maximum of 720 converter units). The flexible switch design uses any-to-any wavelength converters, which make the switch fabric more expensive.

Also from Fig. 9, we can see that the static mapping switch design is simpler and can provide comparable improvements as the strict node-sharing design. Both designs

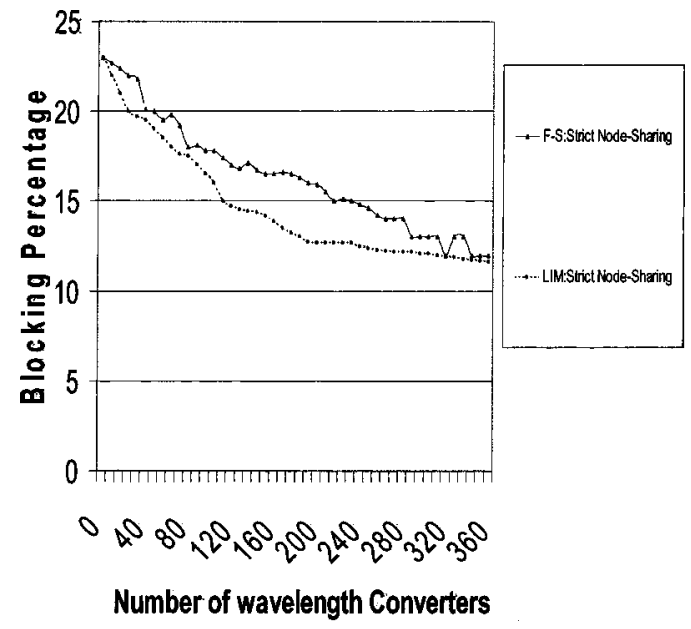

Fig. 7 U.S. Long-Haul network using strict node sharing: LIMITED versus $F$-SEARCH (50 Erlangs, $W=8$ ). 


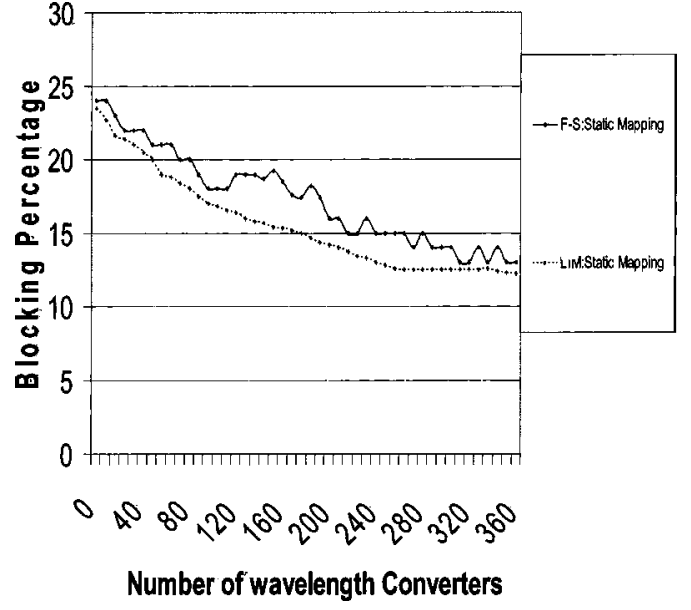

Fig. 8 U.S. Long-Haul network using static mapping: LIMITED versus $F$-SEARCH (50 Erlangs, $W=8$ ).

use a simple wavelength converter with fixed wavelength output (any-to- $\lambda$ converter). When more than $50 \%$ of the maximum possible conversion units are to be placed, all switch designs tend to have comparable performances.

So far we assumed that the traffic is uniformly distributed between node pairs and our placement scheme $k$-MDS assumes that all nodes generate the same traffic in the network. In the next section, we apply the weighted $k$-MDS, referred to as $k$-WMDS, for the NSFNET topology and show its benefits for placing limited WC.

\section{Results Under Nonuniform Traffic}

We consider the NSFNET topology (described earlier in Fig. 4) under nonuniformly distributed traffic between node pairs. We use $k$-WMDS (Ref. 12), which differs from $k$-MDS in the computation of $\operatorname{Connect}_{0}(v)$. Under nonuniform traffic, $\operatorname{Connect}_{0}(v)$ is the product of $\operatorname{Degree}(v)$ and the weight of the node $v$ :

$\operatorname{Connect}_{0}(v)=\operatorname{Degree}(v) \times \operatorname{Weight}(v)$.

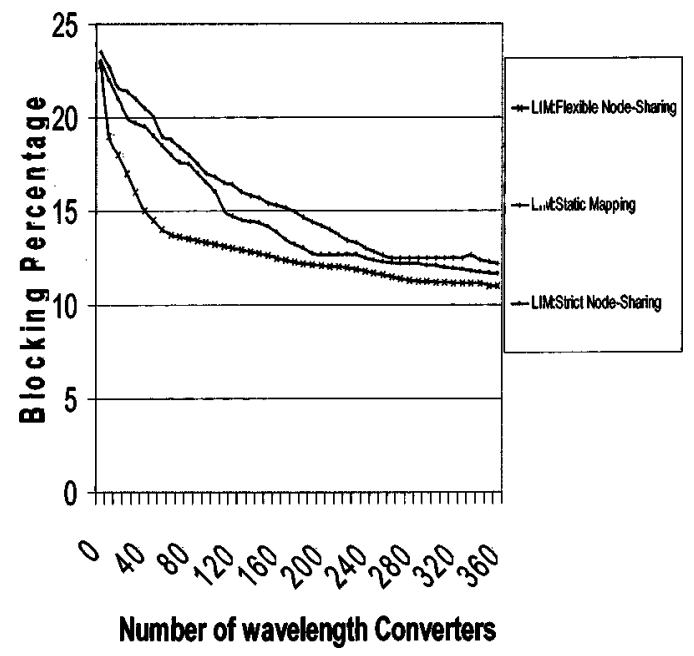

Fig. 9 U.S. Long-Haul network using LIMITED (50 Erlangs, $W$ $=8)$.
Table 1 Assigned node weights for the NSFNET.

\begin{tabular}{cccc}
\hline \hline Node & Weight & Node & Weight \\
\hline 0 & 6 & 8 & 7 \\
1 & 12 & 9 & 2 \\
2 & 7 & 10 & 7 \\
3 & 12 & 11 & 15 \\
4 & 5 & 12 & 3 \\
5 & 8 & 13 & 15 \\
6 & 1 & 14 & 9 \\
7 & 11 & 15 & 2 \\
\hline \hline
\end{tabular}

We applied our voting algorithm to compute the $k$-WMDS set as an approximation for the WMDS for the NFS backbone. Table 1 lists the randomly generated weights for each node in NSFNET. The $k$-WMDS algorithm provided the following results for the NSFNET:

$$
\begin{aligned}
& 1 \text {-WMDS (NSF) }=\{1,4,5,6,9,11,14\}, \\
& \text { 2-WMDS (NSF) }=\{1,4,9,14\}, \text { and } \\
& 3 \text {-WMDS (NSF) }=\{14\} .
\end{aligned}
$$

With the NSFNET topology, $\Re$ is 3 , and the computed disjoint sets are

$$
\begin{aligned}
& \text { SET } 1=3-\text { MDS }=\{14\}, \\
& \text { SET } 2=(2-M D S)-(3-M D S)=\{1,4,9\}, \\
& \text { SET 3 }=(1-\text { MDS })-(2-\text { MDS })=\{5,6,11\}, \\
& \text { SET } 4=\text { the rest of the nodes, i.e., } 9 \text { nodes. }
\end{aligned}
$$

Our comparison results of LIMITED and $F$-SEARCH under nonuniform traffic are presented in Fig. 10 for the flexible node-sharing switch design, in Fig. 11 for the strict node-sharing switch design, and finally in Fig. 12 for the static mapping design. Under a fixed load of 70, we started with no wavelength conversion and at each step added a unit of conversion following the LIMITED algorithm (see Fig. 13). We continued to do so until we placed $50 \%$ of the maximum number possible of wavelength converters units, 400 in this case.

For the three proposed switch designs, the results show that $F$-SEARCH misexamines some combination of converter placements. The curves of $F$-SEARCH (in Figs. 10, 11, and 12) show that this placement algorithm suffers from a local minimum problem. In comparison, the LIMITED

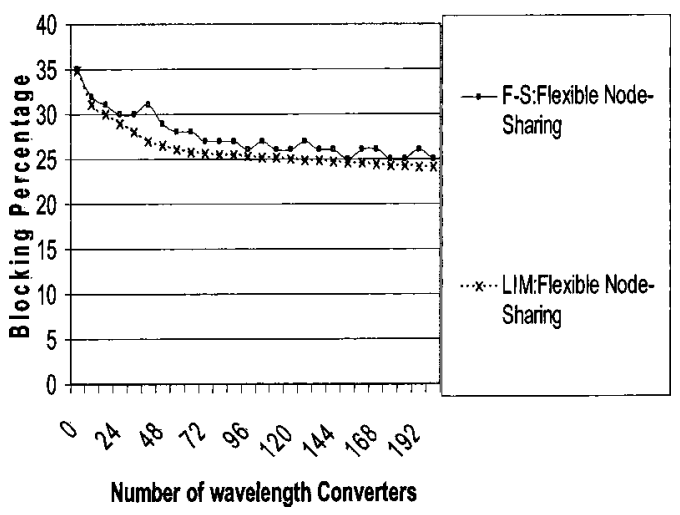

Fig. 10 NSFNET using flexible node sharing: LIMITED versus $F$-SEARCH (70 Erlangs, $W=8$ ). 


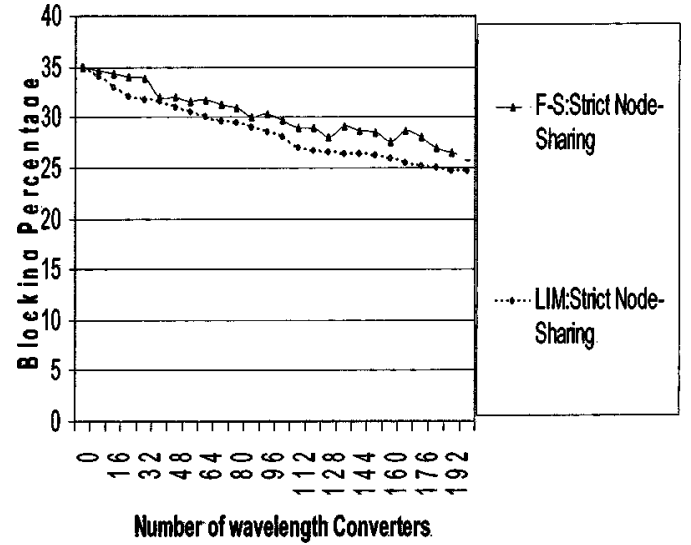

Fig. 11 NSFNET using strict node sharing: LIMITED versus $F$-SEARCH (70 Erlangs, $W=8$ ).

algorithm is more stable since we base our search on $k$-WMDS sets, and it is also faster and less complex.

The cost drives the decision making concerning which switch design to adopt. Static mapping with a cost-effective switch fabric can achieve comparable improvement compared to the strict node-sharing design. This is due to the fact that the strict node sharing enables converters, when idle, to be shared between links. This increases sharing of the WC capability inside the node. The flexible node sharing enables the maximum sharing with its any-to-any wavelength converter units. When few wavelength converters are to be placed, the benefits of the flexible node-sharing switch design are very promising compared to the other two.

The flexible node-sharing design is suitable for networks where the fiber capacity (dense network) or the size of the $\mathrm{OXC}$ is overdesigned for future expansions with unused ports. The static mapping switch design is suited for sparse network with high number wavelength converters to be placed in the network.

\section{Conclusions}

Our proposed optical switch design take advantage of limited WC, including the reduced cost, the improved fault

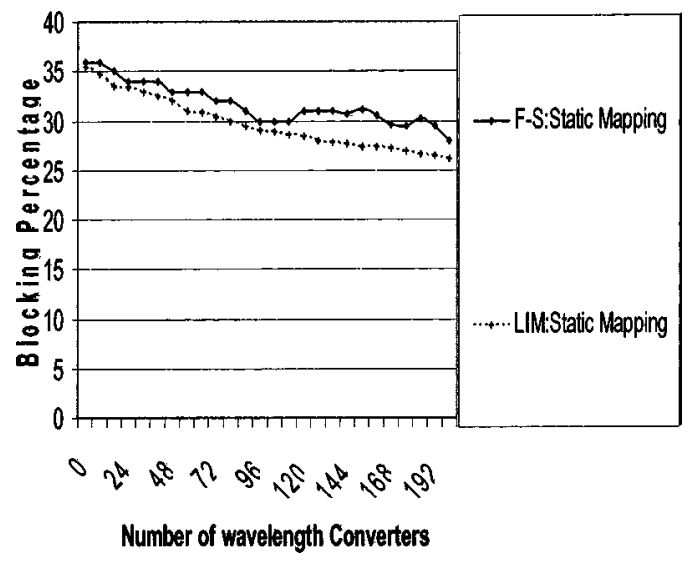

Fig. 12 NSFNET using static mapping: LIMITED versus $F$-SEARCH (70 Erlangs, $W=8$ ).

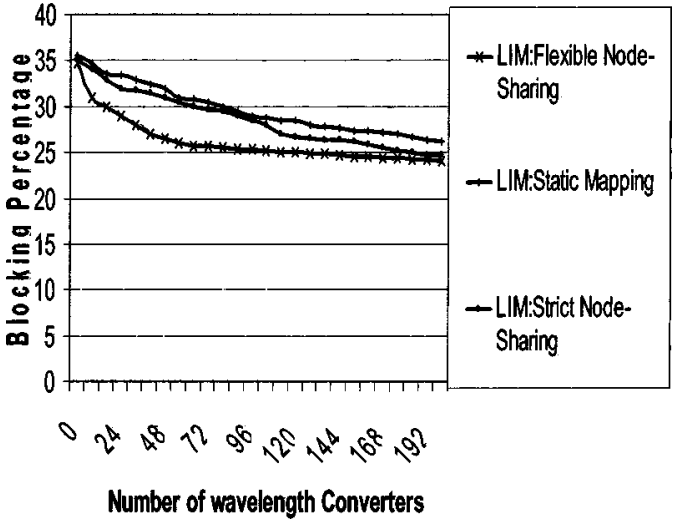

Fig. 13 NSFNET using LIMITED (70 Erlangs, $W=8$ ).

tolerance, and the ability to provide nearly similar improvement as full WC in selected nodes of the network. Our results also show that the limited conversion capability can achieve performance very close to that of the full conversion capability, while not only decreasing the optical switch cost but also enhancing its fault tolerance.

The decision concerning the switch design to use depends on the number of wavelength converters to be placed and the traffic load in the network. When $25 \%$ or fewer WC units are available in the network, the flexible node sharing is recommended as the optical switch design of choice. When more than half of the maximum wavelength converters are to be placed, all three designs seem to have comparable performances. On the other hand, the static mapping switch design is recommended when more than $50 \%$ of the maximum possible number are placed in the network. This is due to the cost and simplicity of the static mapping switch design.

Initially, the network traffic was assumed to be uniformly distributed on the node pairs. We investigated an extension of $k$-MDS for nonuniform traffic, referred to as $k$-WMDS, for realistic networks exploitation, as in Ref. 12. Our model assigns weights to each node based on the generated traffic and we use a WMDS scheme ( $k$-WMDS) for the placement of WC under nonuniform traffic.

Our assumption in this paper is that all nodes have the same optical switch design. In future work, we will investigate the combination of three designs in a network where certain nodes can have a flexible node-sharing switch design and other might have static mapping or strict nodesharing switch designs.

\section{Acknowledgments}

This work has been supported by the National Science Foundation (NSF) under Grant No. 0114418 and Grant No. 9980316.

\section{References}

1. S. Subramaniam, M. Azizoglu, and A. K. Somani, "All-optical networks with sparse wavelength conversion," IEEE/ACM Trans. Network 4, 544-557 (1996).

2. K. R. Venugopal, E. E. Rajan, and P. S. Kumar, "Performance analysis of wavelength converters in WDM wavelength routed optical networks," in Proc. 5th Int. Conf. on High Performance Computing, pp. 239-246 (1998)

3. J. Iness and B. Mukherjee, "Sparse wavelength conversion in 
wavelength-routed WDM optical networks," Photon. Net. Commun. 1(3), pp. 183-205 (1999)

4. M. Sivakumar and S. Subramaniam, "On the performance impact of wavelength assignment and wavelength conversion architecture and placement algorithms," Opt. Net. Mag. 3(2), 44-53 (2002).

5. K. Lee and $\mathrm{V}$. Li, "Routing and switching in a wavelength convertible optical network," in Proc. IEEE INFOCOM, pp. 578-585 (1993).

6. K. Lee and V. Li, "A wavelength convertible optical network," $J$. Lightwave Technol. 11, 962-970 (1993).

7. A. S. Arora and S. Subramaniam, "Wavelength conversion placement in WDM mesh optical networks," Photon. Net. Commun. 4(2), 167177 (2002).

8. D. Li and X. Jia, "Allocating wavelength converters in shared-perlink structure in WDM networks," IEE Proc.-Commun. 149(3), 185188 (2002)

9. M. El Houmaidi, M. Bassiouni, and G. Li, "Dominating set algorithms for sparse placement of full and limited wavelength converters in WDM optical networks," J. Opt. Network. 2(6), 162-177 (2003).

10. R. Karp, "Reducibility among combinatorial problems," in Complex ity of Computer Computations, R. Miller and J. Thatcher, Eds., pp. 85-103. Plenum Press, New York (1972).

11. C. Lund and M. Yannakakis, "On the hardness of approximating minimization problems," J. Assoc. Comput. Mach. 41(5), 960-981 (1994).

12. M. El Houmaidi and M. Bassiouni, " $k$-weighted minimum dominating sets for sparse wavelength converters placement under nonuniform traffic," in Proc. MASCOTS 03, the 11th IEEE/ACM Int. Symp. on Modeling, Analysis and Simulation of Computer and Telecommunication Systems (2003).

13. X. Jia, D. Du, X. Hu, H. Huang, and D. Li, "Placement of wavelength converters for minimal wavelength usage in WDM networks," in Proc. IEEE INFOCOM, Vol. 3, pp. 1425-1431 (2002).

14. G. Wilfong and P. Winkler, "Ring routing and wavelength translation," in Proc. 9th Annu.-ACM-SIAM Symp. on Discrete Algorithms, pp. 333-341 (1998).

15. Y. Wang, L. Li, and S. Wang, "A new algorithm of design protection for wavelength-routed networks and efficient wavelength converter placement," in Proc. ICC 01, IEEE Int. Conf. on Communications, Vol. 6, pp. 1807-1811 (2001).

16. O. Crochat and J. L. Boudec, "Design protection for WDM optical networks," IEEE J. Sel. Areas Commun. 16(7), 1158-1165 (1998).

17. S. Bose, Y. N. Singh, A. N. Raju, and B. Popat, "Sparse converter placement in WDM networks and their dynamic operation using pathmetric based algorithms," in Proc. of ICC 02, IEEE Int. Conf. on Communications, Vol. 5, pp. 2855-2859 (2002).

18. R. Melhem, S. Li, and T. Znati, "Minimizing wavelength conversions in WDM path establishment," J. Photon. Net. Commum. 3(3), 197211 (2001).

19. K. R. Venugopal, M. Shivakumar, and P. S. Kumar, "A heuristic for placement of limited range wavelength converters in all-optical networks," in Proc. IEEE INFO COM, Vol. 2, pp. 908-915 (1999).

20. J. Yates, J. Lacey, D. Everitt, and M. Summerfield, "Limited wavelength translation in all-optical networks," in Proc. INFOCOM, Vol. 3, pp. 954-961 (1996)
21. L. Ruan, D. Du, X. Hu, X. Jia, D. Li, and Z. Sun, "Converter placement supporting broadcast in WDM optical networks," IEEE Trans. Comput. 50(7), 750-758 (2001).

Mounire El Houmaidi received his MSc degree in computer science from the University of Central Florida and is currently pursuing his $\mathrm{PhD}$ degree in computer science with an emphasis on optical networking. He was recently awarded National Science Foundation (NSF) graduate fellowship under the Integrated Graduate Education and Research Training (IGERT) Program. His research interests include optical networks, network management, and wireless communication.

Mostafa A. Bassiouni received his BSc and MSc degrees in computer science from Alexandria University and received his $\mathrm{PhD}$ degree in computer science from the Pennsylvania State University in 1982. He is currently a professor of computer science at the University of Central Florida, Orlando. His research interests include distributed systems, computer networks, real-time protocols, and concurrency control. He has authored over 140 papers published in various computer journals, book chapters, and conference proceedings. His research has been supported by grants from the Army Research Office (ARO), the Advanced Research Projects Agency (ARPA), the National Science Foundation (NSF), the Simulation Training, and Instrumentation Command (STRICOM), Project Manager Training Devices (PM-TRADE), the Cincinnati Bell Information Systems Certification Program (CBIS), Harris, and the State of Florida. He was a member of the program committee of several conferences, the program committee chair of CSMA'98 and CSMA'2000, and the guest coeditor of a special issue of the Journal of Simulation Practice and Theory.

Guifang $\mathbf{L i}$ is an associate professor of optics and electrical and computer engineering at the University of Central Florida. He received his $\mathrm{PhD}$ degree in electrical engineering from the University of Wisconsin at Madison. His research interests include all-optical signal processing for optical networks, fiber-radio systems, rf photonics, and nonlinear dynamics. He received the NSF Presidential Young Investigator award and the Office of Naval Research Young Investigator award. He currently directs the National Science Foundation (NSF) Integrated Graduate Education and Research Training (IGERT) Program in optical communications and networking. He is an associate editor of Optical Networks. 